ARTICLE

Received 18 Sep 2013 | Accepted 26 Nov 2013 | Published 2 Jan 2014

DOI: $10.1038 /$ ncomms 4011

\title{
Room-temperature sub-band gap optoelectronic response of hyperdoped silicon
}

Jonathan P. Mailoa', Austin J. Akey' ${ }^{1}$, Christie B. Simmons', David Hutchinson², Jay Mathews ${ }^{3}$, Joseph T. Sullivan ${ }^{1}$, Daniel Recht ${ }^{4}$, Mark T. Winkler ${ }^{1} \dagger$, James S. Williams ${ }^{5}$, Jeffrey M. Warrender ${ }^{3}$, Peter D. Persans ${ }^{2}$, Michael J. Aziz ${ }^{4} \&$ Tonio Buonassisi ${ }^{1}$

Room-temperature infrared sub-band gap photoresponse in silicon is of interest for telecommunications, imaging and solid-state energy conversion. Attempts to induce infrared response in silicon largely centred on combining the modification of its electronic structure via controlled defect formation (for example, vacancies and dislocations) with waveguide coupling, or integration with foreign materials. Impurity-mediated sub-band gap photoresponse in silicon is an alternative to these methods but it has only been studied at low temperature. Here we demonstrate impurity-mediated room-temperature sub-band gap photoresponse in single-crystal silicon-based planar photodiodes. A rapid and repeatable laser-based hyperdoping method incorporates supersaturated gold dopant concentrations on the order of $10^{20} \mathrm{~cm}^{-3}$ into a single-crystal surface layer $\sim 150 \mathrm{~nm}$ thin. We demonstrate room-temperature silicon spectral response extending to wavelengths as long as 2,200 nm, with response increasing monotonically with supersaturated gold dopant concentration. This hyperdoping approach offers a possible path to tunable, broadband infrared imaging using silicon at room temperature.

\footnotetext{
${ }^{1}$ School of Engineering, Massachusetts Institute of Technology, 77 Massachusetts Avenue, Cambridge, Massachusetts 02139, USA. ${ }^{2}$ Department of Physics, Applied Physics, and Astronomy, Rensselaer Polytechnic Institute, 110 8th Street, Troy, New York 12180, USA. ${ }^{3}$ US Army ARDEC - Benét Laboratories, 1 Buffington Street, Watervliet, New York 12189, USA. ${ }^{4}$ Harvard School of Engineering and Applied Sciences, 29 Oxford Street, Cambridge, Massachusetts 02138, USA. ${ }^{5}$ Research School of Physics and Engineering, The Australian National University, Mills Road, Canberra 0200, Australian Capital Territory, Australia. †Present address: IBM Thomas J. Watson Research Center, 1101 Kitchawan Road, Yorktown Heights, New York 10598, USA. Correspondence and requests for materials should be addressed to J.P.M. (email: jpmailoa@alum.mit.edu) or to T.B. (email: buonassisi@mit.edu).
} 
$\mathrm{D}$ eveloping a low-cost broadband infrared detector with array-based real-time imaging capability at room temperature is of interest for telecommunications, security, energy and research and development applications ${ }^{1-5}$. Siliconbased detectors satisfy the low-cost and on-chip complementary metal-oxide semiconductor (CMOS) compatibility criteria, but their infrared photoresponse is fundamentally limited by the 1.12-eV band gap $(\lambda=1,110 \mathrm{~nm})$. Previous attempts to extend the photoresponse of silicon-based devices into the short-wavelength infrared regime $(\lambda=1,400-3,000 \mathrm{~nm})$ at room temperature focused on forming heterostructures with $\mathrm{SiGe}$ alloys $^{6-8}$ or microstructures of thermally absorbing material ${ }^{9,10}$, and modifying the intrinsic band structure via intentional introduction of defects ${ }^{11-17}$. Growing microstructures of foreign materials on top of silicon results in processing complexities that can compromise CMOS compatibility ${ }^{18,99}$. Furthermore, integration of thermally absorbing materials can be effective for short-wavelength infrared response but results in slow pixel response times intrinsically limited by the thermal time constant, which hinders applications in array-based real-time imaging systems at room temperature ${ }^{10}$.

The direct modification of silicon's electronic band structure is a promising approach for imaging applications, but significant advances on room-temperature sub-band gap photoresponse in silicon to date are mostly focused on fiber-coupled singlepoint detector applications. Published approaches predominantly involve intentionally damaging the silicon lattice ${ }^{12-17}$, which produces a measurable sub-band gap absorption coefficient $\left(\alpha=2-4 \mathrm{~cm}^{-1}\right)^{16}$ compared with untreated silicon $\left(\alpha<2 \times 10^{-7} \mathrm{~cm}^{-1} \text { for } \lambda>1,400 \mathrm{~nm}\right)^{20}$. To increase the optical path length and sub-band gap absorptance, these devices are integrated with micron-sized waveguide resonators ${ }^{13,17}$. By intentionally damaging the silicon lattice, Geis et al. ${ }^{16}$ fabricated single-point, fibre-coupled waveguide photodiodes and phototransistors with room-temperature sub-band gap $(\lambda=1,550 \mathrm{~nm})$ photoresponse of 20 and $50 \mathrm{AW}^{-1}$, respectively ${ }^{16,17}$. These results correspond to impressive quantum efficiencies of 16 and 40 , much larger than unity because of avalanche gain ${ }^{16,17}$. Unfortunately, the waveguide architecture renders this device responsive for only a narrow band of wavelengths, and the requirement for coplanar illumination impedes incorporation into imaging arrays.

Another approach involves incorporating chalcogen dopants into silicon through picosecond or femtosecond laser irradiation. This approach has resulted in a limited number of reports of infrared photorespons $\mathrm{e}^{21,22}$. However, the origin of the sub-band gap photoresponse is not well understood or controlled owing to the complexity of the laser-processed microstructure involving structural defects and multiple chemical phase ${ }^{23}$. To elucidate the origin of sub-band gap photoresponse in chalcogen-doped silicon, sulphur ions have been implanted into silicon and the resulting amorphous layer subjected to pulsed laser melting (PLM)-induced rapid solidification using a nanosecond laser, resulting in a single-crystalline silicon layer with sulphur concentrations above $10^{19} \mathrm{~cm}^{-3}$ (ref. 24). This nanosecond laser-hyperdoping process is repeatable and yields homogenous and single-crystal material, reducing the material's complexity compared with the microstructure with multiple phases found in silicon irradiated with picosecond or femtosecond lasers ${ }^{23}$. However, there are no reports of room-temperature sub-band gap photoresponse for this single-crystalline material. As chalcogen hyperdoping introduces only donor states into the silicon band gap, the resulting material has a large backgroundfree carrier concentration at room-temperature ${ }^{25,26}$. This large background-free carrier concentration overwhelms the sub-band gap photoconductivity signal, making the observation of room- temperature sub-band gap photoconductivity in chalgogenhyperdoped silicon difficult ${ }^{27}$. Similar problems have been encountered in titanium-hyperdoped silicon for which sub-band gap photoconductivity is observed only at liquid nitrogen temperature or below ${ }^{28}$. This is similar to prior work on impurity-diffused silicon photodetectors where the demonstration of sub-band gap photoresponse has been limited to low temperatures 29,30 . Room-temperature sub-band gap photoresponse of hyperdoped single-crystalline silicon remains elusive.

In this study, we report room-temperature sub-band gap photoresponse from single-crystalline silicon hyperdoped with an alternative dopant, gold, to concentrations as high as $5 \times 10^{20} \mathrm{~cm}^{-3}$. We present planar photodiode devices suitable for imaging-array applications fabricated from silicon hyperdoped with gold (Si:Au). Steady-state and transient photoresponse measurements demonstrate that the photodiode optoelectronic response extends well into the sub-band gap regime of silicon, to at least $\lambda=2,200 \mathrm{~nm}$. Furthermore, the magnitude of the response increases monotonically with gold dopant concentration. The homogenous, single-crystalline nature of $\mathrm{Si}: \mathrm{Au}$ gives insight into the likely physical mechanism of enhanced sub-band gap photoresponse, a discrete set of Au-induced donor and acceptor mid-gap energy levels that generate free carriers in response to infrared light, yet reduce background-free carrier concentrations via self-compensation. As PLM induces substantial heating only in the top $150 \mathrm{~nm}$ of the wafer, it might be added as the last step of any CMOS-compatible optoelectronic device fabrication process in user-defined device areas, limiting potential unintentional contamination effects of $\mathrm{Au}$.

\section{Results}

Gold hyperdoping of silicon. The top $150 \mathrm{~nm}$ of three identical $<100>n$-type silicon wafers were implanted with gold ions using an implantation energy of $50 \mathrm{keV}$ and doses of $3 \times 10^{14}$, $7 \times 10^{14}$ and $1 \times 10^{15} \mathrm{~cm}^{-2}$. PLM was used to melt the amorphous implanted region, which rapidly solidifies back into singlecrystal silicon with high, non-equilibrium dopant concentration (Fig. 1a) ${ }^{24}$. Because of gold's high diffusive velocity and segregation coefficient in liquid silicon ${ }^{31}$, the high solidification speed outpaces the kinetics of gold segregation and results in a supersaturated, single-crystalline solid solution ${ }^{31,32}$. An Nd:YAG nanosecond pulse was used to melt and rapidly solidify the gold-hyperdoped silicon (Si:Au) region, and in situ timeresolved reflectivity ${ }^{33}$ measurements showed a melt duration of 25-30 ns, corresponding to a solidification speed of about 7$10 \mathrm{~m} \mathrm{~s}^{-1}$ based on a dopant diffusion simulation in established literature ${ }^{31}$. This process is represented pictorially in Fig. 1a; more detail about the PLM process can be found in the Methods section.

After PLM, secondary ion mass spectrometry (SIMS) was performed to measure the gold atomic concentration as a function of depth (Fig. 1b). The solid solubility limit of gold in silicon at room temperature is estimated to be below $10^{15} \mathrm{~cm}^{-3}$ (refs 34-37). Supersaturated gold concentrations were achieved up to $150 \mathrm{~nm}$ depth for all three gold doses, with the largest gold concentration peak of $5 \times 10^{20} \mathrm{~cm}^{-3}$ measured within the top $10 \mathrm{~nm}$ of the Si:Au layer. This corresponds to 1 at.\% Au concentration, more than four orders of magnitude above the room-temperature equilibrium solid solubility limit of gold in silicon. High-resolution transmission electron microscopic measurements confirmed that the entire Si:Au layer was single crystalline, without formation of any extended defects, secondary phases or cellular breakdown (Fig. 1b) ${ }^{38}$. Because of the large concentration of mid-gap dopant levels introduced by the gold impurities, the hyperdoped layer absorbs sub-band gap light as 
a

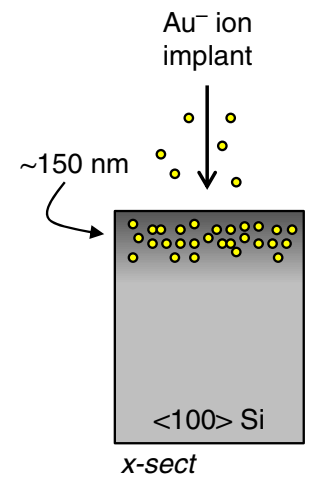

Pulsed

laser melting
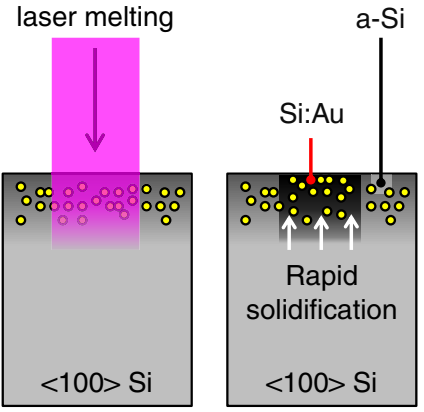

b Protective Si:Au

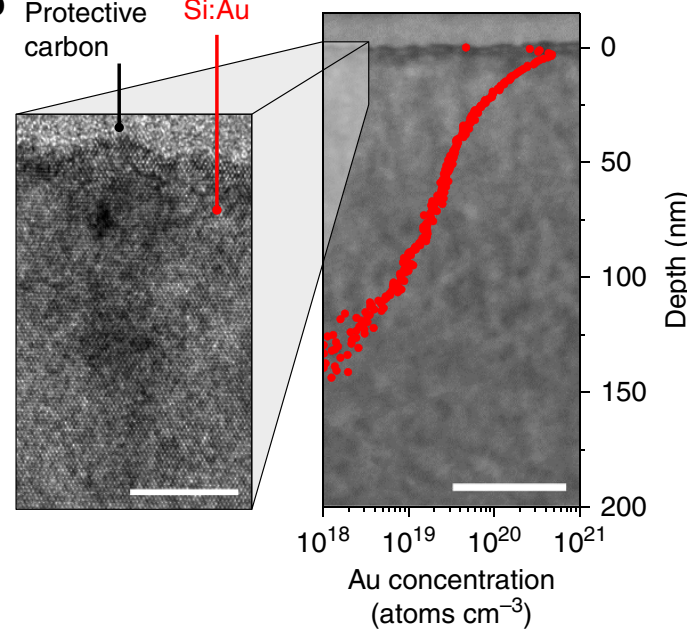

Figure 1 | Fabrication of Si:Au. (a) Fabrication of Si:Au: ion implantation of Au followed by PLM. Rapid solidification follows the PLM process, producing single-crystalline Si:Au on the top $150 \mathrm{~nm}$ of the silicon wafer. The silicon area not melted by the PLM remains amorphous. (b) SIMS profile of Si:Au with $1 \times 10^{15} \mathrm{~cm}^{-2}$ gold implantation dose, showing that the gold concentration in the Si:Au is more than four orders of magnitudes beyond the solid solubility limit of gold in silicon. The transmission electron microscopic images show that for this gold implantation dose, the entire Si:Au layer is crystalline. Scale bar, $10 \mathrm{~nm}$ (left) and $50 \mathrm{~nm}$ (right).

shown in the infrared transmission image through the sample taken using an InGaAs camera (Fig. 2a). Transmission (T) and reflection $(R)$ were measured using ultraviolet-visible-nearinfrared spectrophotometry to quantify the sub-band gap absorptance $(A=1-T-R)$ in the hyperdoped silicon. Similar to chalcogen-hyperdoped silicon ${ }^{39}$, sub-band gap light absorptance increases with increasing $\mathrm{Au}$ concentration (Fig. 2b). Approximating the sub-band gap absorptance occurring uniformly throughout the hyperdoped region $(150 \mathrm{~nm}$ thickness), the average sub-band gap absorption coefficient $\alpha$ at $\lambda=1,550 \mathrm{~nm}$ of the Si:Au with the highest gold dose is estimated to be about $600 \mathrm{~cm}^{-1}(1 \%$ absorptance averaged over a $150-\mathrm{nm}$ thick layer; see Methods), which is larger than that of germanium at the same wavelength $\left(\sim 300 \mathrm{~cm}^{-1}\right)^{40}$.

Steady-state room-temperature sub-band gap photoresponse. To measure the room-temperature sub-band gap photoresponse in $\mathrm{Si}: \mathrm{Au}$, photodiodes were fabricated (see Methods). The dark $I-V$ curves (Fig. $3 \mathrm{~b}$ ) for photodiodes fabricated on $n$-type silicon substrates exhibit rectification behaviour, as expected when the majority carriers in supersaturated $\mathrm{Si}: \mathrm{Au}$ are holes ${ }^{41}$. In contrast, $\mathrm{Si} \mathrm{Au}$ on a $p$-type $\mathrm{Si}$ substrate does not show rectification (Supplementary Fig. 1). Reactive ion etching and electron-beam $\mathrm{Ti} / \mathrm{Au}$ contact evaporation were used to electrically isolate and contact a $1 \times 1 \mathrm{~mm}^{2}$ (ref. 2) Si:Au area to define the active area of the device. An identical photodiode was fabricated on the same substrate but with the Si:Au layer replaced by boron-doped silicon (Si:B) as a reference sample (Supplementary Fig. 2).

The photodiodes were then connected to a comparative resistor, a lock-in amplifier and a voltage source $\left(V_{\mathrm{IN}}=-5 \mathrm{~V}\right)$ that placed the devices under reverse bias (Fig. 3a). A diode laser (optically chopped at frequency $f=714 \mathrm{~Hz}$ ) was focused to a $100-150-\mu \mathrm{m}$ spot size on the photodiode surface. The comparative resistor value of $R=1 \mathrm{k} \Omega$ was chosen such that the reverse bias voltage across the photodiodes was close to $5 \mathrm{~V}\left(I_{\text {dark }} R<<\left|V_{\text {in }}\right|\right)$. The laser spot was then scanned across the photodiode surface. Sub-band gap optoelectronic response was observed only in the $1 \times 1-\mathrm{mm}^{2}$ area of the photodiode corresponding to the electrically connected $\mathrm{Si}$ :Au region.

A map of sub-band gap response at $\lambda=1,550 \mathrm{~nm}$ is shown in Fig. 3d for a sample with implantation dose of $10^{15} \mathrm{Au} \mathrm{cm}^{-2}$. a
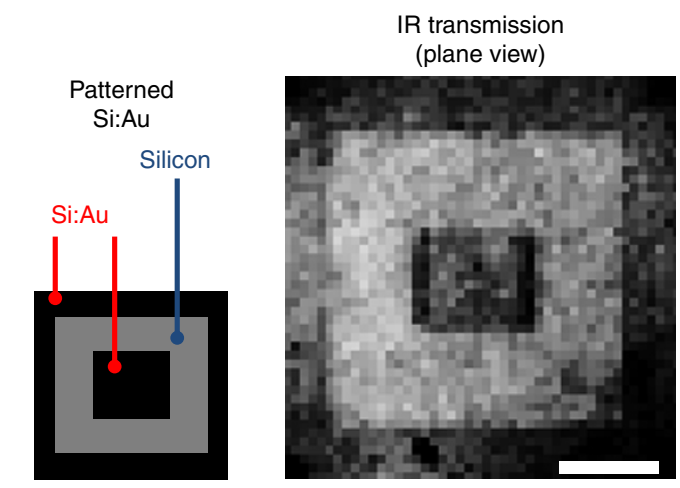

b

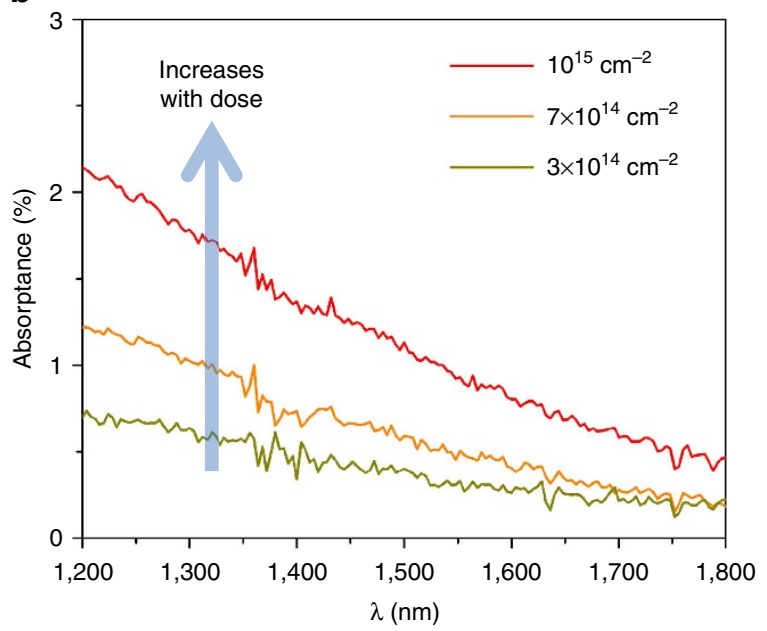

Figure 2 | Sub-band gap absorptance of Si:Au. (a) Transmission of subband gap light through patterned Si:Au taken using an InGaAs camera. Light transmission is reduced by absorption in Si:Au (dark areas) relative to the crystalline silicon (bright areas). Scale bar, $1 \mathrm{~mm}$. (b) Sub-band gap light absorptance (1-transmission-reflection) of Si:Au for various gold implantation dose relative to the sub-band gap light absorptance in the reference plain silicon sample, measured using ultraviolet-visible-nearinfrared spectrophotometry. Sub-band gap light absorptance increases with increasing gold dose. 
a

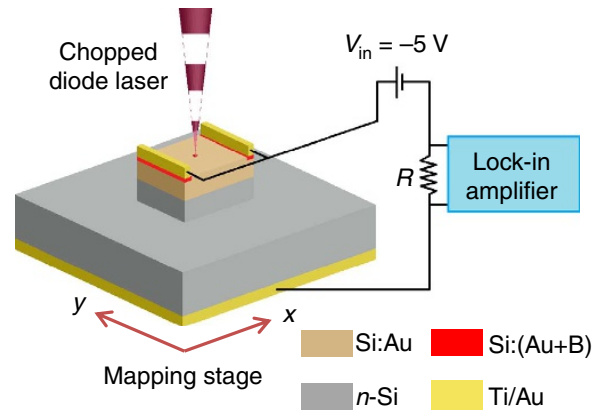

b

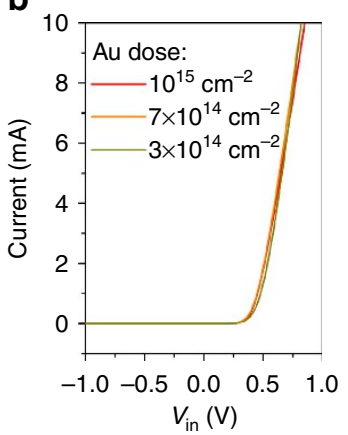

C

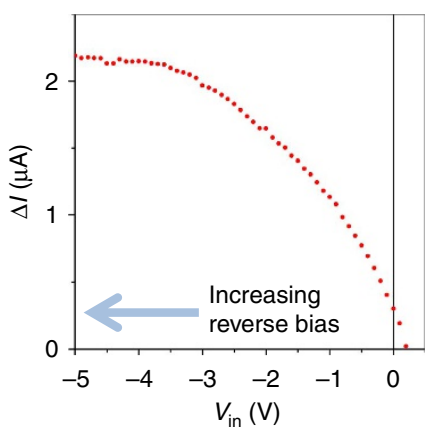

d

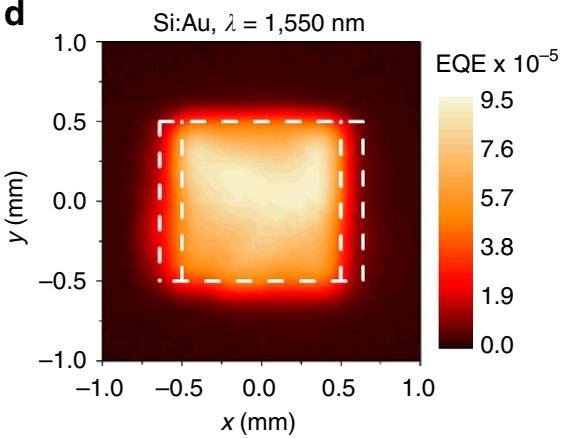

e

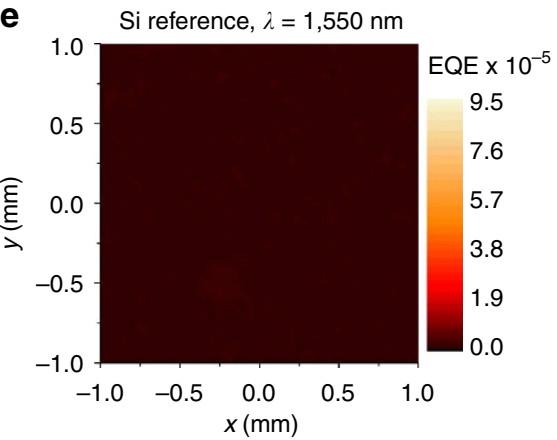

f

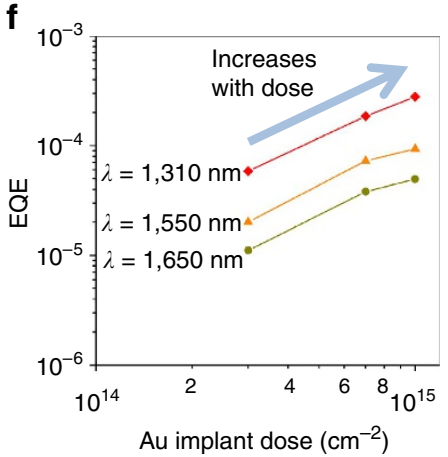

Figure 3 | Room-temperature sub-band gap photoresponse in Si:Au. (a) Si:Au photodiode with Si:Au layer on n-Si substrate operating at reverse bias. The optically chopped diode laser light is focused to $100-150 \mu \mathrm{m}$ spot size and is scanned across the photodiode surface. (b) The dark I-V curve of Si:Au on $n$-type Si shows rectification behaviour for gold implantation dose of $3 \times 10^{14}-1 \times 10^{15} \mathrm{~cm}^{-2}$, indicating that the majority carriers in Si:Au are holes. (c) The difference between dark and illuminated $I-V$ of the photodiode with $10^{15} \mathrm{~cm}^{-2}$ gold dose. Increasing reverse bias across the junction increased carrier drift velocity in the Si:Au layer, improving the collection of photo carriers excited by the sub-band gap light. (d) Mapped external quantum efficiency (EQE) showing a $1 \times 1 \mathrm{~mm}^{2}$ Si:Au active area for $\lambda=1,550 \mathrm{~nm}$ and gold implantation dose of $10^{15} \mathrm{~cm}^{-2}$, confirming the Si:Au layer as the sole source of the sub-band gap photoresponse in the photodiode. (e) No sub-band gap response is observed when the Si:Au is replaced with Si:B in the reference silicon photodiode. (f) EQE for various sub-band gap wavelengths increases with the gold concentration in the Si:Au photodiode.

The spatial uniformity of the photoresponse within the $1 \times 1-\mathrm{mm}^{2}$ area demonstrates that internal photoemission from the metal contact electrodes is not the dominant cause of the sub-band gap photoresponse $\mathrm{e}^{42}$. The reference Si:B photodiode control samples did not show any measurable sub-band gap optoelectronic response (Fig. 3e), further confirming that the Si:Au layer is the origin of the room-temperature sub-band gap photoresponse. This observation is in contrast with the low-temperature subband gap photoresponse previously reported in titaniumhyperdoped silicon, where the sub-band gap photoresponse of the reference crystalline silicon is reported to be larger than that of the titanium-hyperdoped silicon ${ }^{43}$. The sub-band gap photoresponse measurements on the Si:Au photodiodes under $5 \mathrm{~V}$ reverse bias were performed with different sub-band gap wavelengths $(\lambda=1,310,1,550$ and $1,650 \mathrm{~nm})$; spatially optimized external quantum efficiency (EQE) measurements for sub-band gap wavelengths for all $\mathrm{Au}$ doses are shown in Fig. 3f. Si:Au photodiode response increases monotonically with increasing gold concentration for all three sub-band gap wavelengths, with the maximum EQE of $2.8 \times 10^{-4}$ and $9.3 \times 10^{-5}$ for $\lambda=1,310$ and $1,550 \mathrm{~nm}$, respectively. More detail about the EQE calculation can be found in the Methods section.

Room-temperature spectral response of $\mathrm{Si} A \mathrm{Au}$ photodiodes. The extent and mechanism of $\mathrm{Si}: \mathrm{Au}$ room-temperature optoelectronic spectral response was further studied via transient photoresponse measurements. Nanosecond pulses with tunable wavelengths $(\lambda=1,100-2,300 \mathrm{~nm})$ were derived from an optical parametric amplifier $(\mathrm{OPA})^{44}$ pumped by a frequency-tripled,
Q-switched Nd:YAG laser. The pulses were passed through a long pass filter and a Pellin Broca prism to eliminate any undesired wavelengths (for example, laser harmonics) before incidence $n$ the photodiode. The shape of the spectral responses for the Si:Au photodiode with $1 \times 10^{15} \mathrm{~cm}^{-2}$ implant dose and the reference $\mathrm{Si}: \mathrm{B}$ photodiode are shown in Fig. 4a. Because of the high intensity of the sub-band gap laser pulse, a sub-band gap photoresponse signal appeared within the reference silicon sample owing to two-photon absorption (TPA $)^{45}$. This signal responded quadratically with pulse intensity, as expected for a two-photon process, and could be reduced by tuning the pulse intensity appropriately. The spectral response in the reference sample agrees well with the calculated value expected for TPA, $I(\lambda) \beta(\lambda)$ where $I(\lambda)$ is the intensity of the pulse laser used and $\beta(\lambda)$ is the published value of two-photon absorption coefficient ${ }^{45}$ for each wavelength (see Methods for the calculation of TPA).

Figure $4 \mathrm{~b}$ shows the strength of the photoresponse signal with respect to the applied laser pulse intensity for both above gap and sub-band gap light. The photoresponse increased linearly with increasing laser pulse intensity except for the sub-band gap photoresponse in the reference silicon sample, which increased quadratically with increasing laser intensity due to TPA. In addition to the different scaling with photon intensity, the TPA signal was easily distinguished from the photoconductive signal because the transient decay of the response was much slower; this characteristic should be expected for TPA because free carriers were generated and had to be collected from the entire silicon substrate. To mitigate the effect of TPA from both Si:Au and reference silicon spectral response in Fig. $4 \mathrm{a}$, only the shortlived peak of the transient signal was used in the definition 
a

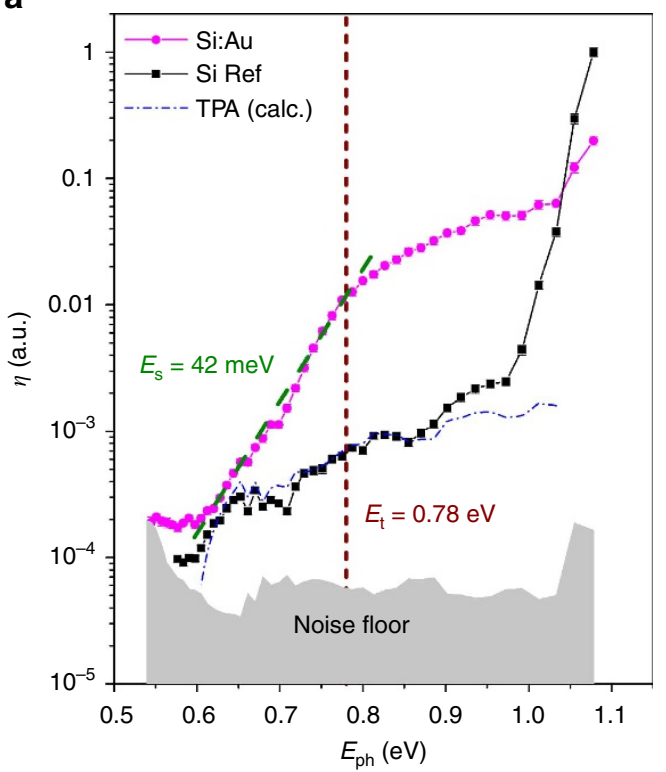

b
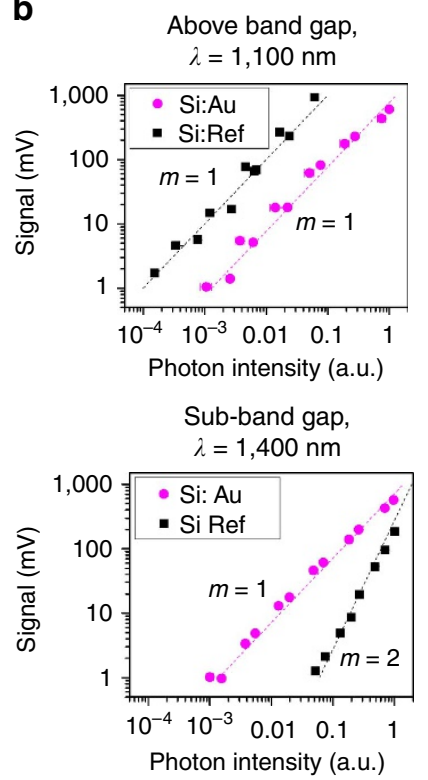

C

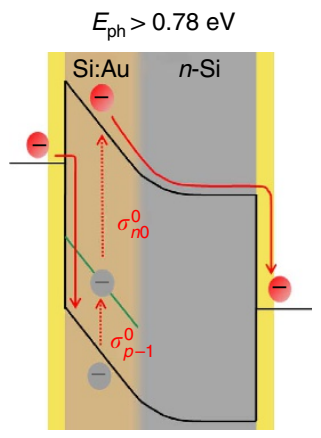

d

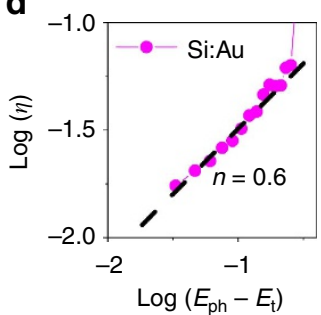

Figure 4 | Sub-band gap photoresponse mechanism of Si:Au. (a) Si:Au spectral response $\eta$ as a function of photon energy measured using tunable wavelength transient photoresponse measured at room temperature for the photodiode with the highest gold dose of $10^{15} \mathrm{~cm}^{-2}$. A kink in the spectral response is observed at the threshold energy $E_{\mathrm{t}}=0.78 \mathrm{eV}$, which corresponds to the substitutional gold donor level in silicon. For $E_{\mathrm{ph}}<E_{\mathrm{t}}$, the response is characterized by the Urbach absorption edge with a slope of $E_{\mathrm{s}}=42 \mathrm{meV}$. (b) Above-band gap and sub-band gap photoresponse of the Si:Au and reference silicon photodiodes for varying photon intensity, confirming that the sub-band gap response in the reference silicon is caused by two-photon absorption (TPA) in the substrate. Under standard operating conditions ( $100 \mathrm{~W} \mathrm{~cm}^{-2}$ or lower), no measurable TPA was observed in the reference silicon photodiode. (c) Possible sub-band gap photoresponse mechanism for substitutional gold in silicon through the donor level for $E_{\mathrm{ph}}>0.78 \mathrm{eV}$. (d) The polynomial dependence $(n=0.6)$ of $\eta$ on $E_{\mathrm{ph}}$, for $E_{\mathrm{ph}}$ close to $E_{\mathrm{t}}\left(0.8 \mathrm{eV}<E_{\mathrm{ph}}<0.94 \mathrm{eV}\right)$, suggesting that the defect-state wavefunction is close to that of a delta function.

of the dimensionless figure of merit $\eta=A \cdot \Delta I_{\text {peak }} / N_{\text {ph }}$, where $A=4.8 \times 10^{21} \mathrm{~A}^{-1}$ is a normalization constant, $\Delta I_{\text {peak }}$ is the photo-generated current and $N_{\mathrm{ph}}$ is the number of photons in the laser pulse. We observe measureable spectral response of Si:Au extending to $E_{\mathrm{ph}}=0.55 \mathrm{eV}$, and a distinct change in slope at photon energy $E_{\mathrm{ph}}=0.78 \mathrm{eV}$ (Fig. 4a). This later spectral feature appears to be dopant specific, as discussed in the next section.

\section{Discussion}

We first comment on the probable origins of the sub-band gap photoresponse of Si:Au. Previous work by Sah et al. ${ }^{46}$ and Okuyama et al. ${ }^{48}$ utilized temperature-dependent photoconductivity and spectrally resolved low-temperature photoconductivity, respectively, to determine the energy level of gold impurities in silicon diffused with gold concentration below the solid solubility limit. At these low dopant concentrations, the $\mathrm{Au}$-induced defect states are single, discrete levels with noninteracting localized states. In that study ${ }^{48}$, the reported spectral response has a distinct change in slope near the photon energy $E_{\mathrm{ph}}=0.78 \mathrm{eV}$, which corresponds to the substitutional gold donor energy level in silicon. In our study, the same spectral feature was observed in the Si:Au photodiode with a gold dose of $1 \times 10^{15} \mathrm{~cm}^{-2}$, suggesting that the sub-band gap photoresponse mechanism in these Si:Au photodiodes may be the same as that in gold-diffused silicon measured at liquid nitrogen temperature ${ }^{48}$. The higher gold concentration achieved by our PLM process apparently enables one to observe this effect at roomtemperature.

Next, we address the role of 'drift' in carrier extraction from the Si:Au layer. It is known that deep-level traps such as $\mathrm{Au}$ in silicon reduce the electronic carrier lifetime through non-radiative recombination ${ }^{49}$. With the gold concentration of $N_{\mathrm{t}}=10^{20} \mathrm{~cm}^{-3}$ and the saturated electron velocity of $v_{\mathrm{s}}=10^{7} \mathrm{~cm} \mathrm{~s}^{-1}$ assumed in the depleted Si:Au layer ${ }^{50}$, an electron trapping rate $\tau_{\mathrm{eo}}=\left(\sigma_{\mathrm{n}}^{\mathrm{t}} v_{\mathrm{s}} N_{\mathrm{t}}\right)^{-1}=5$ ps would be expected, given an electron capture cross-section $\sigma_{\mathrm{n}}^{\mathrm{t}}=2 \times 10^{-16} \mathrm{~cm}^{2}$ of gold in silicon ${ }^{51}$. Despite the low minority-carrier lifetime in Si:Au, some free electrons excited by the sub-band gap light in the hyperdoped region were able to escape into the $n$-type substrate. This process is aided because the Si:Au layer is fully depleted because of the low carrier concentration in gold-doped silicon (see Methods and Supplementary Fig. 3). This low carrier concentration in the Si:Au layer is believed to be caused by the self-compensation due to the ionization of deep acceptor and donor trap levels of substitutional gold in silicon ${ }^{41,52,53}$. The resulting full depletion of the Si:Au causes the majority of the applied reverse bias voltage to drop across this layer, thereby facilitating carrier transport via drift.

This behaviour is in contrast with past work in sulphurhyperdoped silicon ${ }^{25}$, where the free carrier concentration is between $10^{17}$ and $10^{20} \mathrm{~cm}^{-3}$ and the minority-carrier transport in the hyperdoped region is driven by the comparatively weaker process of diffusion ${ }^{27}$. The application of $5 \mathrm{~V}$ reverse bias across the hyperdoped layer appears sufficient to saturate the photoresponse. This is shown in Fig. 3c, where the photogenerated current produced by $\lambda=1,550 \mathrm{~nm}$ light saturates with increasing reverse bias voltage. While higher gold concentration is expected to reduce the sub-band gap photoresponse by reducing the minority-carrier lifetime, the applied reverse bias voltage seems to be capable of overcoming the negative effect of higher gold concentration. Hence, the sub-band gap optoelectronic response increases with the increasing sub-band gap absorptance in samples with higher gold concentrations (Fig. 3f).

Next, we discuss how the dopant type affects the infrared spectral response. In this dopant-mediated, extrinsic sub-band 
gap photoresponse mechanism, electrons can be excited into the conduction band through the dopant state(s). One probable mechanism is the electron excitation from the valence band to the conduction band through the donor energy level, which has previously been shown for gold-diffused silicon at low temperature (Fig. 4c) ${ }^{48,54}$. In ref. 28 , donor-assisted transition arises at threshold energy of $0.78 \mathrm{eV}$. Inspecting Fig. $4 \mathrm{a}$, similar threshold energy is evident in the Si:Au photodiode. For photon energy $E_{\mathrm{ph}}$ larger than the threshold energy $E_{\mathrm{t}}=0.78 \mathrm{eV}$ (ref. 34), the transitions characterized by photoionization cross-section $\sigma_{p-1}^{0}$ (from valence band to the donor state) and $\sigma_{n 0}^{0}$ (from donor state to the conduction band) are both possible. Electrons from the valence band can then be excited into the conduction band and swept away by the strong reverse bias electric field across the $\mathrm{Si}: A u$ layer into the $n$-type substrate. They are then transported as majority carriers in the conduction band before being extracted by the back contact. However, for $E_{\mathrm{ph}}<E_{\mathrm{t}}$ the transition from the donor level to the conduction band characterized by $\sigma_{n 0}^{0}$ is turned off; thus, electrons excited into the donor level eventually recombine into the valence band without leaving the Si:Auhyperdoped region. As the donor-level/conduction-band transition is unavailable, the magnitude of the sub-band gap response for the energy range $E_{\mathrm{ph}}<E_{\mathrm{t}}$ becomes exponentially less likely with decreasing excitation energy. One possible model for this exponential decay is the dependence $\eta\left(E_{\mathrm{ph}}\right) \propto \exp \left(\left(E_{\mathrm{ph}}-E_{\mathrm{t}}\right) / E_{\mathrm{s}}\right)$ where $E_{\mathrm{s}}$ is the slope of the Urbach absorption edge due to disorder in solids ${ }^{55} . E_{\mathrm{s}}=42 \mathrm{meV}$ has been extracted from fits to the experimental data as the slope of the Urbach edge. This is larger than $k T=26 \mathrm{meV}$, suggesting that there is a disorderinduced broadening in either the impurity states or the conduction band edge of Si:Au. The spectral kink at $E_{\mathrm{ph}}=0.78 \mathrm{eV}$ closely matches the spectral response of golddiffused silicon at low temperature, suggesting that it should be possible to tune the sub-band gap spectral response of hyperdoped silicon by using alternative dopant elements that introduce levels at different energies ${ }^{48}$.

Further analysis can be done on the spectral response of $\mathrm{Si}: \mathrm{Au}$ for $E_{\mathrm{ph}}>0.78 \mathrm{eV}$. Various models have been proposed to explain the dependence of the photoionization cross-section $\sigma\left(E_{\mathrm{ph}}\right)$ for deep levels in semiconductor. If the deep-level wavefunction is a localized state with delta function like spatial dependence in the length scale of the atomic radius, for $E_{\mathrm{ph}}$ close to the threshold energy $E_{\mathrm{t}}=0.78 \mathrm{eV}$ the approximate dependence $\sigma\left(E_{\mathrm{ph}}\right) \propto\left(E_{\mathrm{ph}}-E_{\mathrm{t}}\right)^{1 / 2}$ is expected ${ }^{56,57}$. In contrast, for a state with hydrogenic like wavefunction the approximate dependence $\sigma\left(E_{\mathrm{ph}}\right) \propto\left(E_{\mathrm{ph}}-E_{\mathrm{t}}\right)^{3 / 2}$ is expected ${ }^{58}$. The conversion efficiency $\eta$ is directly proportional to the photoionization cross-section in the $\mathrm{Si}: \mathrm{Au}$ photodiode, and as such the dependence $\eta\left(E_{\mathrm{ph}}\right) \propto\left(E_{\mathrm{ph}}-E_{\mathrm{t}}\right)^{n}$ for $E_{\mathrm{ph}}$ close to $E_{\mathrm{t}}=0.78 \mathrm{eV}$ can be used to infer the spatial behaviour of the deep-level wavefunction. The range for the fit is set to $0.8 \mathrm{eV}<E_{\mathrm{ph}}<0.94 \mathrm{eV}$ to reduce the influence of the Urbach edge, and the fit is shown in Fig. 4d. The polynomial dependence of $n=0.6$ is extracted, suggesting that the deep-level state wavefunction for the donor level of Si:Au has a spatial dependence much closer to that of a delta function ${ }^{56,57}$ than that of a hydrogenic wavefunction ${ }^{58}$.

As the first demonstration devices, there is room to improve the absolute room-temperature sub-band gap EQE of $\sim 10^{-4}$. Two focus areas should include improving sub-band gap light absorptance and incorporating best-in-class photodiode-design practices to enhance carrier collection. This can be achieved, for example, by using a larger gold implantation dose for the PLM process and applying an anti-reflection coating on the photodiode to increase the sub-band gap absorptance of the Si:Au layer beyond its current $\sim 1 \%$ absorptance. Increasing the thickness of the Si:Au layer could also increase the optical path length and sub-band gap absorptance. Further gains may be possible via optimization of device design: because most of the gold dopants are concentrated near the silicon surface (Fig. 1b), the majority of free carriers generated by sub-band gap light are believed to be within the top $20 \mathrm{~nm}$ of the Si:Au surface. As the surfacelimited lifetime for $W=20 \mathrm{~nm}$ thick silicon is in the order of $\tau_{\mathrm{s}}=W / S=2$ ps when a non-passivated surface recombination velocity $S=10^{6} \mathrm{~cm} \mathrm{~s}^{-1}$ is assumed ${ }^{59}$, the sub-band gap photoresponse in $\mathrm{Si} A \mathrm{Au}$ with a bulk lifetime of $\sim 5 \mathrm{ps}$ is prone to surface recombination. Incorporating surface passivation could potentially suppress this recombination activity. We conservatively estimate that practical application of these improvements may increase the room temperature sub-band gap optoelectronic response of $\mathrm{Si}: \mathrm{Au}$ photodiodes by two orders of magnitudes to $\mathrm{EQE}=10^{-2}$, although no physical limits are known at this time to prevent even higher EQE.

In summary, we report the room-temperature sub-band gap optoelectronic response in silicon hyperdoped with gold using a two-step process: ion implantation followed by PLM. The subband gap optoelectronic response is shown to correspond to known gold dopant energy levels in silicon and increase with the implanted gold concentration. This work represents a fundamentally new approach to achieve sub-band gap optoelectronic response in silicon that avoids structural defects and interface management issues associated with combining foreign materials and silicon. While the magnitude of the room-temperature subband gap response demonstrated here is in the $\mathrm{EQE} \sim 10^{-4}$ range, further improvements are likely via device architecture optimization. The planar, single-crystal nature of the hyperdoped silicon layer created using the PLM process makes this an attractive material candidate for room-temperature sub-band gap photon-imaging devices based on silicon.

\section{Methods}

Nanosecond PLM. Silicon wafers $(<100>, n$-type, resistivity $\sim 3 \Omega \mathrm{cm}$, minoritycarrier lifetime $\sim 180 \mu \mathrm{s}$ ) were ion-implanted with ${ }^{197} \mathrm{Au}^{-}$at an energy of $50 \mathrm{keV}$, to doses of $3 \times 10^{14}, 7 \times 10^{14}$ or $1 \times 10^{15}$ atoms $\mathrm{cm}^{-2}$. The wafers were then pulsed-laser melted by a single pulse from an Nd:YAG laser, using the third harmonic wavelength of $355 \mathrm{~nm}$, with a pulse duration full-width-half-maximum (FWHM) of $5 \mathrm{~ns}$ and a fluence of $0.7 \mathrm{~J} \mathrm{~cm}^{-2}$. The resulting material was characterized by SIMS using a Physical Electronics 6650 Dynamic SIMS instrument Operating conditions were the following: $6 \mathrm{keV} \mathrm{Cs}$ ion beam at $1 \mathrm{nA}$, with SIMS craters being $50 \mu \mathrm{m}^{2}$; depth was measured ex situ by contact profilometry. $\mathrm{Au}$ concentrations were calibrated against known ion-implantation doses from the as-implanted regions of each sample, normalized by the ${ }^{28} \mathrm{Si}$ signal. Transmission electron microscopic samples were fabricated by focused-ion beam milling and liftout; transmission electron microscopic imaging was performed on a JEOL 2100 high-resolution transmission electron microscope operated at $200 \mathrm{keV}$ in bright-field mode.

Approximation of sub-band gap $\alpha$ in Si:Au. We model the Si:Au as a uniform $t=150 \mathrm{~nm}$ thin film on top of crystalline silicon wafer. It is assumed that the real refractive index of $\mathrm{Si}: \mathrm{Au}$ is not any different from that of a normal crystalline silicon. Furthermore, to simplify the model, it is assumed that light interference in the thin-film Si:Au region does not play a critical role in the light propagation. As crystalline silicon does not absorb sub-band gap light, the sub-band gap light absorption in the Si:Au layer can then be approximated as:

$$
\begin{aligned}
A & =(1-R) \alpha t+(1-R) \alpha t(1-\alpha t) R+(1-R) \alpha t(1-\alpha t)^{2} R^{2}+\ldots \\
& =\frac{(1-R) \alpha t}{1-(1-\alpha t) R}
\end{aligned}
$$

where $A$ is the sub-band gap absorption measured using the spectrophotometer and $R$ is the fraction of light reflected when light propagates through the air-silicon interface. Taking the value of $R \sim 0.36$ and $A \sim 1 \%, \alpha=A(1-R) / t(1-R-A R)=$ $600 \mathrm{~cm}^{-1}$ can be approximated. This is the lower bound for the value of $\alpha$ in Si:Au with gold implantation dose of $10^{15} \mathrm{~cm}^{-2}$. In reality, $90 \%$ of the gold atoms are buried within the top $20 \mathrm{~nm}$ of the Si:Au layer. As the majority of the sub-band gap light absorption is believed to occur within this $20 \mathrm{~nm}$ thin layer, the actual value of $\alpha$ in the region with highest gold concentration may be several times larger. 
Si:Au photodiode-type determination. As we have detailed in the main text, at the supersaturated concentrations obtained for Si:Au implanted with gold doses of $3 \times 10^{14}, 7 \times 10^{14}$ and $1 \times 10^{15} \mathrm{~cm}^{-2}$, the majority carriers in Si:Au are holes. When Si:Au is fabricated on an $n$-type silicon substrate, the resulting structure is a junction that shows the rectifying behaviour of a diode. In contrast, when Si:Au is fabricated on a $p$-type silicon substrate, the resulting structure does not rectify. A dark $I-V$ curve for one such structure is shown in Supplementary Fig. 1, where Si:Au with gold implantation dose of $10^{14} \mathrm{~cm}^{-2}$ was fabricated on a $p$-type substrate.

Photodiode device fabrication. See Supplementary Fig. 2 for device dimensions. Before the PLM process, boron ions were selectively implanted into the gold-doped silicon area under the top contacts to improve contact resistance. The selected area for the boron implantation was patterned using photolithography, and ${ }^{11} \mathrm{~B}^{+}$ions $\left(3 \times 10^{14} \mathrm{~cm}^{-2}\right.$ dose, $2 \mathrm{keV}$ implantation energy) were implanted into the top $30 \mathrm{~nm}$ of the gold-doped area. The entire sample then underwent the PLM process (described above), creating a homogenous single-crystal Si:Au layer with a pair of rectangular $\mathrm{Si}:(\mathrm{Au}+\mathrm{B})$-patterned areas positioned for subsequent contact deposition. The depth of the boron doping is simulated to be $\sim 70 \mathrm{~nm}$ deep after the PLM process. Photolithography and $\mathrm{SF}_{6}$ reactive ion etch were then used to create a $1-\mu \mathrm{m}$-deep trench that isolated the $1 \times 1 \mathrm{~mm}^{2}$ rectangular Si:Au and the $\mathrm{Si}:(\mathrm{Au}+\mathrm{B})$ contact area from the rest of the Si:Au layer. Photolithography, $e$-beam evaporation and lift-off were used to deposit $30 / 160 \mathrm{~nm}$ of Ti/Au contacts on top of the Si:(Au + B) areas. On the back of the silicon wafer, a uniform 30/160 nm Ti/Au layer was deposited across the entire surface as the photodiode back contact. The back contact was attached to an external electrode by conductive silver paste, while the top contacts were connected to electrodes by Al wire bonds. For the reference silicon photodiode, the same boron-doping profile is used everywhere instead of $\mathrm{Si}: \mathrm{Au}$, creating a $p-n$ photodiode without any Au-hyperdoped silicon.

EQE calculation. A 2-V peak-to-peak square wave $S(t)$ can be expressed as $S(t)=1.273 \sin (\omega t)+0.4244 \sin (3 \omega t)+0.2546 \sin (\omega t)+\ldots$ where $\omega$ is the angular frequency and $t$ is time. As the optically chopped light can be approximated as a square wave and the lock-in amplifier output $V_{\text {out }}$ is the root mean squared value of the first Fourier component of the signal, the mapped EQE for each coordinate on the photodiode can be calculated using the formula $\operatorname{EQE}(x, y)=2.22 V_{\text {out }}(x, y) /$ $\operatorname{Rq} \phi(\lambda)$, where $V_{\text {out }}$ is the signal output from the lock-in amplifier, $R$ is the value of the comparative resistor, $q$ is the electron charge and $\phi(\lambda)$ is the number of photons incident on the sample per unit time measured using a calibrated germanium photodiode. The Si:Au photodiodes were operated at $-5 \mathrm{~V}$ reverse bias for these EQE measurements.

Modelling of fully depleted hyperdoped region in Si:Au diode. When $I-V$ responses of Si:Au photodiodes were measured under large reverse biases, it is observed that the dark current increases almost linearly with the increase of reverse bias voltage. This is in contrast to that of a non-depleted diode that normally approach a steady dark current following the diode equation $I=I_{o}\left(e^{q V / k T}-1\right)$. A simple diode model to simulate this behaviour is developed in the PC1D device simulator. A $p$-type emitter layer with a thickness of $150 \mathrm{~nm}$ and a low carrier lifetime of $20 \mathrm{ps}$ is used to represent the Si:Au layer. The base is represented with an $n$-type silicon with $500 \mu \mathrm{m}$ thickness and $180 \mu$ s carrier lifetime (parameter obtained from the actual substrate). PC1D simulation shows that the hyperdoped Si:Au region is indeed fully depleted, and that the resulting dark $I-V$ curve shows the same linear behaviour for large reverse bias voltages (Supplementary Fig. 3).

Transient-pulse laser photoresponse. The laser pulse with $10 \mathrm{~ns}$ FWHM exiting the OPA consisted of the sub-band gap signal wavelength $\left(\lambda_{\mathrm{s}}\right)$, the idler wavelength $\left(\lambda_{\mathrm{i}}\right)$, as well as possible leakage of other above-band gap wavelengths $\left(\lambda_{\mathrm{a}}\right)$ due to the inner working of the OPA. $\lambda_{\mathrm{a}}$ was first filtered out using a razor-edge $1,064 \mathrm{~nm}$ long pass edge filter (Edmund Optics, NT47-510). The resulting beam was further purified by dispersing it using Pellin Broca prism mounted on a rotation stage and selecting only the desired wavelength $\left(\lambda_{s}\right)$ with a subsequent aperture. The rotation stage was calibrated such that across the entire spectral range considered only $\lambda_{\mathrm{s}}$ could illuminate the sample. Infrared neutral density filters mounted on a filter wheel were then used to tune the beam intensity. The intensity was chosen such that the magnitude of the transient voltage was much smaller than the supply voltage $V_{\text {in }}=-5 \mathrm{~V}$ to prevent signal saturation. Furthermore, the beam intensity was also chosen such that the signal due to two-photon absorption (TPA) in the substrate was minimized. An average of 200 pulses was used for the acquisition of each data point.

TPA model for reference photodiode sub-band gap response. One of the characteristics of two-photon absorption (TPA) is characterized by the sub-band gap absorption coefficient $\alpha_{\mathrm{TPA}}(\lambda)=I(\lambda) \beta(\lambda)$ where $I(\lambda)$ is the intensity of the pulse laser used and $\beta(\lambda)$ is the published value of two-photon absorption coefficient ${ }^{45}$ for each wavelength. The value of $\beta(\lambda)$ for $\lambda=1,200-2,200 \mathrm{~nm}$ is about $1 \mathrm{~cm} \mathrm{GW}^{-1}$. The maximum pulse laser energy in our transient photoresponse experiment is about $100 \mu \mathrm{J}$ per pulse with $\sim 0.5 \mathrm{~cm}$ beam diameter. As the laser pulse FWHM is about $10 \mathrm{~ns}$, the maximum instantaneous intensity of the laser pulses incident on the photodiode samples are $50 \mathrm{~kW} \mathrm{~cm}^{-2}$ In this case, maximum $\alpha_{\mathrm{TPA}}(\lambda)$ of $5 \times 10^{-5} \mathrm{~cm}^{-1}$ can be approximated. This absorption coefficient is very small, and consequently for $L=0.5 \mathrm{~mm}$ thick silicon reference photodiode that we used in our experiment the total sub-band gap light intensity absorbed due to TPA can be approximated as $I_{\mathrm{ABS}}(\lambda) \propto I(\lambda)\left(1-\exp \left(-\alpha_{\mathrm{TPA}}(\lambda) 2 L\right)\right) \propto I^{2}(\lambda) \beta(\lambda) 2 L$. The photoresponse signal observed in the oscilloscope is directly proportional to this value, hence the quadratic dependence of the signal to the photon intensity observed in Fig. 3b. The corresponding value for the dimensionless figure of merit $\eta$ is obtained by dividing the photoresponse signal with the sub-band gap photon illumination. Hence, we have the proportionality relationship $\eta(\lambda)=A I(\lambda) \beta(\lambda)$ to model the two-photon absorption in Fig. 4a, where $A$ is the normalization constant.

\section{References}

1. Zhang, Y. et al. Broadband high photoresponse from pure monolayer graphene photodetector. Nat. Commun. 4, 1811 (2013).

2. Lee, S. J. et al. A monolithically integrated plasmonic infrared quantum dot camera. Nat. Commun. 2, 286 (2011).

3. Liu, X. et al. Bridging the mid-infrared-to-telecom gap with silicon nanophotonic spectral translation. Nat. Photonics 6, 667-671 (2012).

4. Luque, A., Marti, A. \& Stanley, C. Understanding intermediate-band solar cells. Nat. Photonics 6, 146-152 (2012).

5. Luque, A. \& Marti, A. Photovoltaics: towards the intermediate band. Nat Photonics 5, 137-138 (2011).

6. Kang, Y. et al. Monolithic germanium/silicon avalanche photodiodes with $340 \mathrm{GHz}$ gain-bandwidth product. Nat. Photonics 3, 59-63 (2008).

7. Michel, J., Liu, J. \& Kimerling, L. C. High-performance Ge-on-Si photodetectors. Nat. Photonics 4, 527-534 (2010).

8. Wang, G. et al. High quality Ge epitaxial layers in narrow channels on Si (001) substrates. Appl. Phys. Lett. 96, 111903 (2010).

9. Yan, J. et al. Dual-gated bilayer grapheme hot-electron bolometer. Nat. Nanotechnol. 7, 472-478 (2012).

10. Tanaka, A. et al. Infrared focal plane array incorporating silicon IC process compatible bolometer. IEEE T. Electron Dev. 43, 1844-1850 (1996).

11. Casalino, M., Coppola, G., Iodice, M., Rendina, I. \& Sirleto, L. Near-infrared sub-band gap all-silicon photodetectors: state of the art and perspectives. Sensors 10, 10571-10600 (2010).

12. Bradley, J. D. B., Jessop, P. E. \& Knights, A. P. Silicon waveguide-integrated optical power monitor with enhanced sensitivity at $1550 \mathrm{~nm}$. Appl. Phys. Lett. 86, 241103 (2005).

13. Doylend, J. K., Jessop, P. E. \& Knights, A. P. Silicon photonic resonatorenhanced defect-mediated photodiode for sub-bandgap detection. Opt. Express 18, 14671-14678 (2010).

14. Grote, R. R. et al. $10 \mathrm{~Gb} / \mathrm{s}$ error-free operation of all-silicon ion-implantedwaveguide photodiodes at $1.55 \mu \mathrm{m}$. IEEE Photon. Techol. Lett. 25, 67-70 (2013).

15. Geis, M. W. et al. CMOS-compatible all-Si high-speed waveguide photodiodes with high responsivity in near-infrared communication band. IEEE Photon. Technol. Lett. 19, 152-154 (2007).

16. Geis, M. W. et al. All silicon infrared photodiodes: photo response and effects of processing temperature. Opt. Express 15, 16886-16895 (2007).

17. Geis, M. W. et al. Silicon waveguide infrared photodiodes with $>35 \mathrm{GHz}$ bandwidth and phototransistors with $50 \mathrm{AW}-1$ response. Opt. Express 17, 5193-5204 (2009).

18. Park, D. S.-W., Kim, K., Pillans, B. \& Lee, J.-B. Polydimethylsiloxane-based pattern transfer process for the post-IC integration of MEMS onto CMOS chips. J. Micromech. Microeng. 14, 335-340 (2004).

19. Lopez, J. L. et al. Integration of RF-MEMS resonators on submicrometric commercial CMOS technologies. J. Micromech. Microeng. 19, 015002 (2009).

20. Green, M. A. \& Keevers, M. J. Optical properties of intrinsic silicon at $300 \mathrm{~K}$. Prog. Photovoltaics 3, 189-192 (1995).

21. Carey, J. E., Crouch, C. H., Shen, M. \& Mazur, E. Visible and near-infrared responsivity of femtosecond-laser microstructured silicon photodiodes. Opt. Lett. 30, 1773-1775 (2005).

22. Hu, S. et al. Improved photoresponse characteristics in Se-doped Si photodiodes fabricated using picosecond pulsed laser mixing. Semicond. Sci. Technol. 27, 102002 (2012).

23. Smith, M. J. et al. The origins of pressure-induced phase transformations during the surface texturing of silicon using femtosecond laser irradiation. J. Appl. Phys. 112, 083518 (2012).

24. Tabbal, M., Kim, T., Warrender, J. M. \& Aziz, M. J. Formation of single crystal sulfur supersaturated silicon based junctions by pulsed laser melting. J. Vac. Sci. Technol. B 25, 1847-1852 (2007)

25. Winkler, M. T. et al. Insulator-to-metal transition in sulfur-doped silicon. Phys. Rev. Lett. 106, 178701 (2011).

26. Ertekin, E. et al. Insulator-to-metal transition in selenium-hyperdoped silicon: observation and origin. Phys. Rev. Lett. 108, 026401 (2012). 
27. Sullivan, J. T. et al. Methodology for vetting heavily doped semiconductors for intermediate band photovoltaics: a case study in sulfur-hyperdoped silicon. J. Appl. Phys. 114, 103701 (2013).

28. Olea, J. et al. Ruling out the impact of defects on the below band gap photoconductivity of Ti supersaturated Si. J. Appl. Phys. 114, 053110 (2013).

29. Redington, R. W. \& van Heerden, P. J. Doped silicon and germanium photoconductors as targets for infrared television camera tubes. J. Opt. Soc. Am. 49, 997-1001 (1959).

30. Sclar, N. Survey of dopants in silicon for $2-2.7$ and 3-5 $\mu \mathrm{m}$ infrared detector application. Infrared Phys. 17, 71-82 (1976).

31. Recht, D. et al. Supersaturating silicon with transition metals by ion implantation and pulsed laser melting. J. Appl. Phys. 114, 124903 (2013).

32. Priolo, F. et al. Trapping of $\mathrm{Au}$ in Si during pulsed laser irradiation: a comparison with ion beam induced segregation. Appl. Phys. Lett. 53, 2486-2488 (1988).

33. Auston, D. H., Surko, C. M., Venkatesan, T. N. C., Slusher, R. E. \& Golovchenko, J. A. Time-resolved reflectivity of ion-implanted silicon during laser annealing. Appl. Phys. Lett. 33, 437-440 (1978).

34. Graff, K. Metal Impurities in Silicon-Device Fabrication (Springer Publishing Company, 1999).

35. Trumbore, F. A. Solid solubilities of impurity elements in germanium and silicon. Bell Syst. Tech. J. 39, 205-233 (1960).

36. Cagnina, S. F. Enhanced gold solubility effect in heavily n-type silicon. J. Electrochem. Soc. 116, 498-502 (1969).

37. Brown, M., Jones, C. L. \& Willoughby, A. F. W. Solubility of gold in p-type silicon. Solid State Electron. 18, 763-770 (1975).

38. Cullis, A. G. et al. Growth interface breakdown during laser recrystallization from the melt. Appl. Phys. Lett. 38, 642-644 (1981).

39. Sullivan, J. T. et al. Soft X-ray emission spectroscopy studies of the electronic structure of silicon supersaturated with sulfur. Appl. Phys. Lett. 99, 142102 (2011).

40. Dash, W. C. \& Newman, R. Intrinsic optical absorption in single-crystal germanium and silicon at $77^{\circ} \mathrm{K}$ and $300^{\circ} \mathrm{K}$. Phys. Rev. 99, 1151-1155 (1995).

41. Valdinoci, M., Colalongo, L., Pellegrini, A. \& Rudan, M. Analysis of conductivity degradation in gold/platinum-doped silicon. IEEE T. Electron. Dev. 43, 2269-2275 (1996).

42. Perera, A. G. U., Yuan, H. X. \& Francombe, M. H. Homojunction internal photoemission far-infrared detectors: photoresponse performance analysis. J. Appl. Phys. 77, 915-924 (1995).

43. García-Hemme, E. et al. Sub-band gap spectral photo-response analysis of Ti-supersaturated Si. Appl. Phys. Lett. 101, 192101 (2012).

44. Dunn, M. H. \& Ebrahimzadeh, M. Parametric generation of tunable light from continuous-wave to femtosecond pulses. Science 286, 1513-1517 (1999).

45. Bristow, A. D., Rotenberg, N. \& van Driel, H. M. Two-photon absorption and Kerr coefficients of silicon for 850-2200 nm. Appl. Phys. Lett. 90, 191104 (2007).

46. Sah, C. T. \& Tasch, Jr A. F. Precise determination of the multiphonon and photon carrier generation properties using the impurity photovoltaic effect in semiconductors. Phys. Rev. Lett. 19, 69-71 (1967).

47. Sah, C. T., Tasch, Jr A. F. \& Schroder, D. K. Recombination properties of the gold acceptor level in silicon using the impurity photovoltaic effect. Phys. Rev. Lett. 19, 71-72 (1967).

48. Okuyama, M., Matsunaga, N., Chen, J.-W. \& Milnes, A. G. Photoionization cross-sections and energy levels of gold, iron, platinum, silver, and titanium in silicon. J. Electron. Mater. 8, 501-515 (1979).

49. Shockley, W. \& Read, Jr W. T. Statistics of the recombination of holes and electrons. Phys. Rev. 87, 835-842 (1952).

50. Canali, C., Majni, G., Minder, R. \& Ottaviani, G. Electron and hole drift velocity measurements in silicon and their empirical relation to electric field and temperatures. IEEE T. Electron. Dev. 22, 1045-1047 (1975).
51. Sah, C. T., Forbes, L., Rosier, L. L. \& Tasch, Jr A. F. Thermal and optical emission and capture rates and cross sections of electrons and holes at imperfection centers in semiconductors from photo and dark junction current and capacitance measurements. Solid State Electron. 13, 759-788 (1970).

52. Weman, H., Henry, A., Begum, T. \& Monemar, B. Electrical and optical properties of gold-doped n-type silicon. J. Appl. Phys. 65, 137-145 (1989).

53. Bullis, W. M. Properties of Gold in Silicon. Solid State Electron. 9, 143-169 (1996).

54. Braun, S. \& Grimmeiss, H. G. Optical properties of gold acceptor and donor levels in silicon. J. Appl. Phys. 45, 2658-2665 (1974).

55. Kurik, M. V. Urbach rule. Phys. Status Solidi A 8, 9-45 (1971).

56. Grimmeiss, H. G. \& Ledebo, L.-A. Spectral distribution of photoionization cross sections by photoconductivity measurements. J. Appl. Phys. 46, 2155-2162 (1975).

57. Grimmeiss, H. G. \& Lebedo, L.-A. Photo-ionization of deep impurity levels in semiconductors with non-parabolic bands. J. Phys. C Solid State 8, 2615-2626 (1975).

58. Lucovsky, G. On the photoionization of deep impurity centers in semiconductors. Solid State Commun. 3, 299-302 (1965).

59. Yablonovitch, E. \& Gmitter, T. Auger recombination in silicon at low carrier densities. Appl. Phys. Lett. 49, 587-589 (1986).

\section{Acknowledgements}

We thank V. Suntharalingam, C. Leitz and M.W. Geis (Lincoln Laboratory) for helpfu discussions; Z.K. Ren (SMART) for PC1D simulation advice; S. Castellanos (MIT) for the help in taking infrared transmission image; and F. Frankel (MIT) for helpful suggestions regarding graphics. Research at Harvard was supported by the US Army-ARDEC under Contract number W15QKN-07-P-0092 and the US Army Research Office under Contract number W911NF-12-1-0196. Research at the ANU was funded by the Australian Research Council, and we also thank the use of facilities provided by the Australian National Fabrication Facility. Research at MIT was supported by National Science Foundation grant for Energy, Power, and Adaptive Systems under Contract number ECCS-1102050, and in part by the National Science Foundation (NSF) and the Department of Energy (DOE) under NSF CA number EEC-1041895. This work was funded in part by the MIT-KFUPM Center for Clean Water and Energy. This work made use of the MTL and CMSE at MIT supported by NSF award DMR-0819762. This work was performed in part at the Center for Nanoscale Systems (CNS), a member of the National Nanotechnology Infrastructure Network (NNIN), which is supported by the National Science Foundation under NSF Award number ECS-0335765. CNS is a part of Harvard University.

\section{Author contributions}

J.P.M., A.J.A., C.B.S., D.H., J.M. and J.S.W. synthesized the materials, made the devices, built the experimental apparatus and carried out the experiments. All authors analysed the data, with critical inputs from all authors. J.P.M. and T.B. were responsible for experimental design and wrote the manuscript, which incorporates critical input from all authors.

\section{Additional information}

Supplementary Information accompanies this paper at http://www.nature.com/ naturecommunications

Competing financial interests: The authors declare no competing financial interests.

Reprints and permission information is available online at http://npg.nature.com/ reprintsandpermissions/

How to cite this article: Mailoa, J. P. et al. Room-temperature sub-band gap optoelectronic response of hyperdoped silicon. Nat. Commun. 5:3011 doi: 10.1038/ ncomms4011 (2014). 